\title{
Universal and anomalous behavior in the thermalization of strongly interacting harmonically trapped gas mixtures
}

\author{
Francisco Jauffred, ${ }^{1}$ Roberto Onofrio, ${ }^{2,3}$ and Bala Sundaram ${ }^{1}$ \\ ${ }^{1}$ Department of Physics, University of Massachusetts, Boston, MA 02125, USA \\ ${ }^{2}$ Dipartimento di Fisica e Astronomia "Galileo Galilei", Università di Padova, Via Marzolo 8, Padova 35131, Italy \\ ${ }^{3}$ Department of Physics and Astronomy, Dartmouth College, 6127 Wilder Laboratory, Hanover, NH 03755, USA
}

\begin{abstract}
We report on the dynamics of thermalization by extending a generalization of the CaldeiraLeggett model, developed in the context of cold atomic gases confined in a harmonic trap, to higher dimensions. Universal characteristics en route to thermalization which appear to be independent of dimensionality are highlighted, which additionally suggest a scaling analogous to turbulent mixing in fluid dynamics. We then focus on features dependent on dimensionality, with particular regard to the role of angular momentum of the two atomic clouds, having in mind the goal of efficient thermalization between the two species. Finally, by considering asymmetry in species numbers, we find that nonlinear interspecies interactions provide a mode locking mechanism between the majority and minority species, relevant to recent experiments involving Fermi-Bose mixtures in the normal phase.
\end{abstract}

\section{INTRODUCTION}

The study of ultracold atomic gases is a vibrant frontier of atomic physics holding the promise of a controllable environment in which to realize model Hamiltonians of interest in condensed matter physics [1, 2, The controllability includes, among other things, the ability to change the sign and the strength of interaction among the atoms, and equally importantly, the effective dimensionality of the system. This latter relies on trapping at temperatures low enough with respect to the energy quantum of the system in the involved dimension, and allows for the study of phenomena otherwise precluded, or more difficult to observe, in the three-dimensional context (for relevant cases see for instance [3] [5]). Consequently, a significant number of studies have been devoted to developing methods to optimize atomic cooling [6, 7]. In particular, the most widespread technique to achieve deeper degeneracy relies on sympathetically cooling fermions via coupling to a bosonic species, which are easier to cool via evaporative cooling. Theoretically, trying to describe the microscopic mechanism for the thermalization of the two atomic species is more challenging as it appears to fall outside the standard descriptions adopted in condensed matter physics or in fluid dynamics.

Recently, we proposed models which extend the Caldeira-Leggett framework [8 15] to a setting where both species exhibit negligible frequency spreads, mimicking the situation typical for trapping and cooling atomic gases [16]. The model was developed and analysed in the case of one-dimensional systems, for which a simplified yet rich dynamics occurs. However, typical configurations for sympathetic cooling are fully threedimensional, especially in the earlier stages of cooling when no degrees of freedom can be considered as frozen. Therefore generalization of the model presented in [16] is deemed necessary to describe sympathetic cooling in realistic situations necessary to guide possible optimization of experimental protocols. This is particularly important for the full quantum degenerate case involving FermiBose mixtures as, in one-dimension, identical specific heats are expected for both Fermi and Bose gases [17, 18].

In this paper we describe progress made in this direction by analysing the thermalization dynamics during the earlier stage of evaporative cooling in which atoms can be still considered as nondegenerate gases. The paper is organized as follows. In Section II we introduce the full three-dimensional model and metrics which may be used to continuously monitor the thermalization dynamics. These are then used to confirm results obtained earlier [16] in the one-dimensional case as well as to explore universal aspects of the transition from non-equilibrium to equilibrium dynamics. Analogous results are shown in higher dimensions with the initial focus on the isolation of common characteristics which are independent of dimensionality, which provide a generic view of the thermalization process. Later, in Section III, we turn to additional parameters that play a possible role in thermalization in higher dimensions and show that their importance is primarily in the early stages. This naturally leads to the identification of setups for which thermalization may proceed at the fastest rate. Finally, in Section IV we discuss unique features of the nonlinear interaction between the two clouds when there is asymmetry in the atom numbers. Specifically, in the limit of strong interactions, the center of mass oscillation frequency of the minority species is forced to lock onto that of the majority species, a situation relevant to observations in recent experiments. More general considerations and future directions of relevance to experimental trapping schemes conclude the paper.

\section{DIMENSIONALLY INDEPENDENT ASPECTS OF THERMALIZATION}

The core idea discussed in Ref. [16] was the extension of the Caldeira-Leggett model [8 15], as a microscopic de- 
scription of thermalization features, to the atomic physics context. The comparison to the Caldeira-Leggett model here is to its genuinely classical features, i.e. as a model for an external environment capable of describing the relaxation to thermal equilibrium of a target system, for instance a harmonic oscillator or a bistable system. This is successfully achieved in the Caldeira-Leggett model by coupling the targeted system in a linear fashion to an infinite and delocalized ensemble of harmonic oscillators with a continuous and unbounded spectrum of frequencies. These two distinctive features of the model require accommodation in the trapped atom context where there is a privileged point in space, the trap origin (thus breaking translational invariance, on which phonon propagation relies in the original Caldeira-Leggett model), and the atoms oscillate at a well-defined frequency, instead of a continuum of frequencies. A secondary feature is that the numbers of atoms in a trap are considerably smaller than in a typical condensed-matter system, resulting in mesoscopic features that can be related to Caldeira-Leggett models with finite size baths [1924. For the specific case of a monochromatic bath, the Caldeira-Leggett model is recovered if the range of interaction is large enough and the interaction strength is appropriately chosen though, as discussed in detail in [19], thermalization does not occur.

The model developed in Ref [16] considered a classical one-dimensional Hamiltonian, consisting of $N_{b}$ particles constituting the 'bath' and $N_{p}$ 'test' particles, of the form

$$
\begin{aligned}
H_{\mathrm{tot}}= & \sum_{m=1}^{N_{p}}\left(\frac{P_{m}^{2}}{2 M}+\frac{M \Omega^{2} Q_{m}^{2}}{2}\right)+\sum_{n=1}^{N_{b}}\left(\frac{p_{n}^{2}}{2 m}+\frac{m \omega^{2} q_{n}^{2}}{2}\right) \\
& +\gamma_{E} \sum_{m=1}^{N_{p}} \sum_{n=1}^{N_{b}} \exp \left[-\frac{\left(q_{n}-Q_{m}\right)^{2}}{\lambda^{2}}\right],
\end{aligned}
$$

which leads to thermalization of the two species under rather general conditions. Although in Ref. [16] a velocity-dependent interaction Hamiltonian was also considered, here we confine ourselves only to an interaction term acting only in configurational space, which results in a considerable simplification of the dynamics and faster computational speed. The model is readily extended to higher dimensions simply by vectorizing the phase-space variables though, as we will see later, a number of other considerations accompany this generalization. Hamilton's equations corresponding to Eq. 11 are integrated numerically using a variable step algorithm which preserves integrals of motion, such as the total energy and the total angular momentum, to machine precision. The initial conditions are drawn from thermal distributions specified by the starting temperatures of each atomic cloud.

In general, cold atomic gases live in higher dimensions, especially in the classical limit for which freezing of one or two dimensions does not occur except in the case of special, highly nonlinear, trapping potentials. The onedimensional case is particularly simple, and for this reason somewhat misleading, as exemplified by the constant

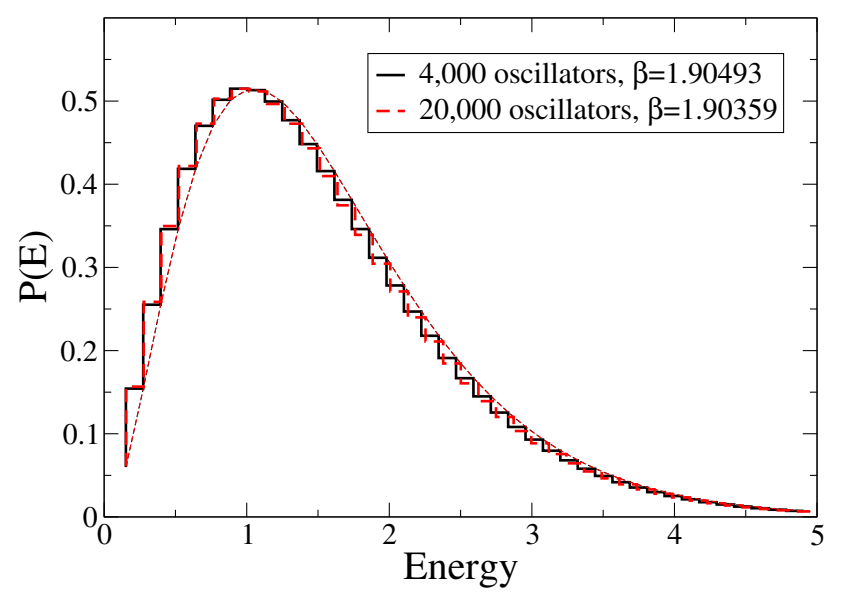

FIG. 1: Energy probability distributions at initial time for $4 \times 10^{3}$ (continuous black histogram) and $2 \times 10^{4}$ (dashed red histogram) harmonic oscillators in $3 \mathrm{D}$ at a nominal inverse temperature of $\beta=2.0$ as set up in the random number generator part of the code for assigning the initial energies for each atom. The inverse temperatures reported in the label arise from a single parameter fit using the probability distribution of Eq. (5), and the two fitting curves (dashed curves) are practically indistinguishable. The inverse temperature resulting from the best fit is systematically smaller by about 5 $\%$ with respect to the predetermined one, a discrepancy due to the coarse graining occurring in binning the distributions. Note that the binning is chosen to be identical in the two situations.

density of states in the limit of non-interacting harmonic oscillators. Consider the case where $N_{p}=1$, if each oscillator of the bath is described by the Hamiltonian $H=p^{2} / 2 m+m \omega^{2} q^{2} / 2$ (therefore neglecting the influence of the $\gamma_{E}$-dependent interaction term $H_{\text {int }}$ as in Eq. (4) of Ref. [16, in a sort of weak-coupling approximation) the phase space surface area of the energy shell $E$ is simply

$$
S=\oint p d q=\pi(2 m E)^{1 / 2}\left(\frac{2 E}{m \omega^{2}}\right)^{1 / 2}=2 \pi \frac{E}{\omega}
$$

This implies that the number of quantum states with energy less than or equal to $E$ is $S /(2 \pi \hbar)=E / \hbar \omega$. The density of states - the number of states per unit of energy - will then be $g(E)=\partial S / \partial E=(\hbar \omega)^{-1}$, i.e. independent of the energy. In general, in D-dimensions the same procedure yields a density of states $g(E)=D E^{D-1} /(\hbar \omega)^{D}$, such that the energy probability distribution will be $P_{D}(E)=\beta^{D} E^{D-1} e^{-\beta E} / \Gamma(D)$, where $\beta$ is the inverse temperature and $\Gamma$ the gamma-function. Specifically, for the interesting cases in $1 \mathrm{D}, 2 \mathrm{D}$, and $3 \mathrm{D}$, we have, respectively 


$$
\begin{aligned}
P_{1}(E) & =\beta e^{-\beta E} \\
P_{2}(E) & =\beta^{2} E e^{-\beta E}, \\
P_{3}(E) & =\frac{1}{2} \beta^{3} E^{2} e^{-\beta E} .
\end{aligned}
$$

As we shall see shortly, these relations can be used to approximately compute $\beta$ over the transition time from non-equilibrium to equilibrium dynamics. Note that deviations from the equilibrium distributions can also be estimated by computing $\beta$ from different combinations of higher moments of the distribution. The consistency of these values is an indication of the appropriateness of the full Boltzmann distribution.

In Fig. 1 we show the energy probability histogram and the related best fit according to the expression in Eq. (5), for two initial configurations differing only in the number of atoms. A comparison between the inverse temperature used for the energy configuration and the value achieved by a best fit to the histogram provides a quantitative assessment of the accuracy of the obtained inverse temperature $\beta$. Figure 1 illustrates that there is no significant improvement in the determination of the inverse temperature with a fivefold increase in the number of particles, with the same binning used for the histograms. This shows, further, that the choice of binning is a greater bottleneck to accurate determinations of the inverse temperature than the size of the sample. From the standpoint of numerics, therefore, dealing with small number of particles $(N=500-1000)$ is sufficient to determine $\beta$ to within a few percent, and within reasonable computational times.

The definitions given above allow also for a practical 'shortcut' to dynamically evaluate the temperature, since the following general expressions hold $\langle E\rangle=D / \beta$, $\left\langle E^{2}\right\rangle=D(D+1) / \beta^{2}$, which lead to the following relationship between the energy variance and the inverse temperature,

$$
\sigma_{E}=\left(\left\langle E^{2}\right\rangle-\langle E\rangle^{2}\right)^{1 / 2}=\frac{\sqrt{D}}{\beta} .
$$

The assessment of the inverse temperature via the simple energy probability densities as in Eq. (6) is only valid, as mentioned above, in the weak coupling limit. This approximation allows for the neglect of the deformation to the ellipsoids, representing the energy surfaces, due to the interaction energy. Ideally, one needs to construct the appropriate constant energy $E$ (including the interaction energy term) surface, count the states within the phase space volume as a function of $E$, and consider the derivative of its dependence. This procedure should then be repeated for different values of $\gamma_{E}$ (and possibly $\lambda$ ). Otherwise only a concept of 'effective' temperature, of limited validity in the strong-coupling limit, must be introduced. It should be noted that that this 'effective temperature' is already used in other contexts [25, 26].
In those studies, the effective $\beta$ serves as a proportionality constant between the rate of change of an inter-species correlation function and a companion response function. We will soon see an analogous relation emerge here as well.

Thermalization was quantified in Ref. [16 by constructing the energy distribution at a set of fixed times and the effective $\beta$ was then determined through fitting to an exponential function. In order to demonstrate that constructing $\beta$ using relation Eq. (6) works equally well to track the thermalization dynamics, we show in Fig. 2(a) the thermalization dynamics using Eq. (6) to compute $\beta(t)$ contrasted with earlier published results in [16. The data agree well and the clear advantage of the continuous tracking afforded by the moment method is seen in the additional details in the thermalization curves, in both the $1 \mathrm{D}$ and the $3 \mathrm{D}$ dynamics shown in Fig. 2(b). The inferences hidden in these details can be extracted on contrasting the relaxation dynamics with the time variation of the total interaction energy. This is shown in Figs. 2(c) and (d) for both 1D and 3D dynamics. The total interaction energy is the sum of all the pairwise interactions according to the last term in Eq. (1) and the large excursions correspond to periodic interactions of sets of particles from the two species. The fact that large numbers of particles are involved is evidenced by the size of the change as contrasted with the value of $\gamma_{E}$. These episodic events are the mechanism for energy transfer and appear correlated with the changes in $\beta$, with the colder species being more sensitive to these interactions. We note here that the interaction term involves pairs of particles each taken from one of the species. As such, it is analogous to the cross-species two-point correlation function whose time variation and accompanying response is used in defining effective temperatures (or $\beta$ ) in describing non-equilibrium systems. The link between the total interaction energy and effective temperature can be motivated by the following argument. Consider the total interaction Hamiltonian

$$
H_{\mathrm{int}}=\gamma_{E} \sum_{m=1}^{N_{t}} \sum_{n=1}^{N_{b}} \exp \left[-\frac{\left(q_{n}-Q_{m}\right)^{2}}{\lambda^{2}}\right]
$$

and its ensemble average

$$
\left\langle E_{\text {int }}\right\rangle=\gamma_{E} N_{b} N_{p} \exp \left[-\frac{\left\langle\left(q_{b}-Q_{p}\right)^{2}\right\rangle}{\lambda^{2}}\right],
$$

where the subscripts indicate a pair of particles drawn from the bath $(b)$ and test particle $(p)$ species and the approximation $<f(x)\rangle=f(<x>)$ is used. We can identify the ensemble average in the exponent as the interspecies two-point correlation function which can be now be written as

$$
C_{b p}=\left\langle\left(q_{b}-Q_{p}\right)^{2}\right\rangle=-\lambda^{2} \ln \left(\frac{\left\langle E_{\text {int }}\right\rangle}{\gamma_{E} N_{b} N_{p}}\right) .
$$

We note here that the correlation between pairs of particles from the two species is a consequence of interaction, 

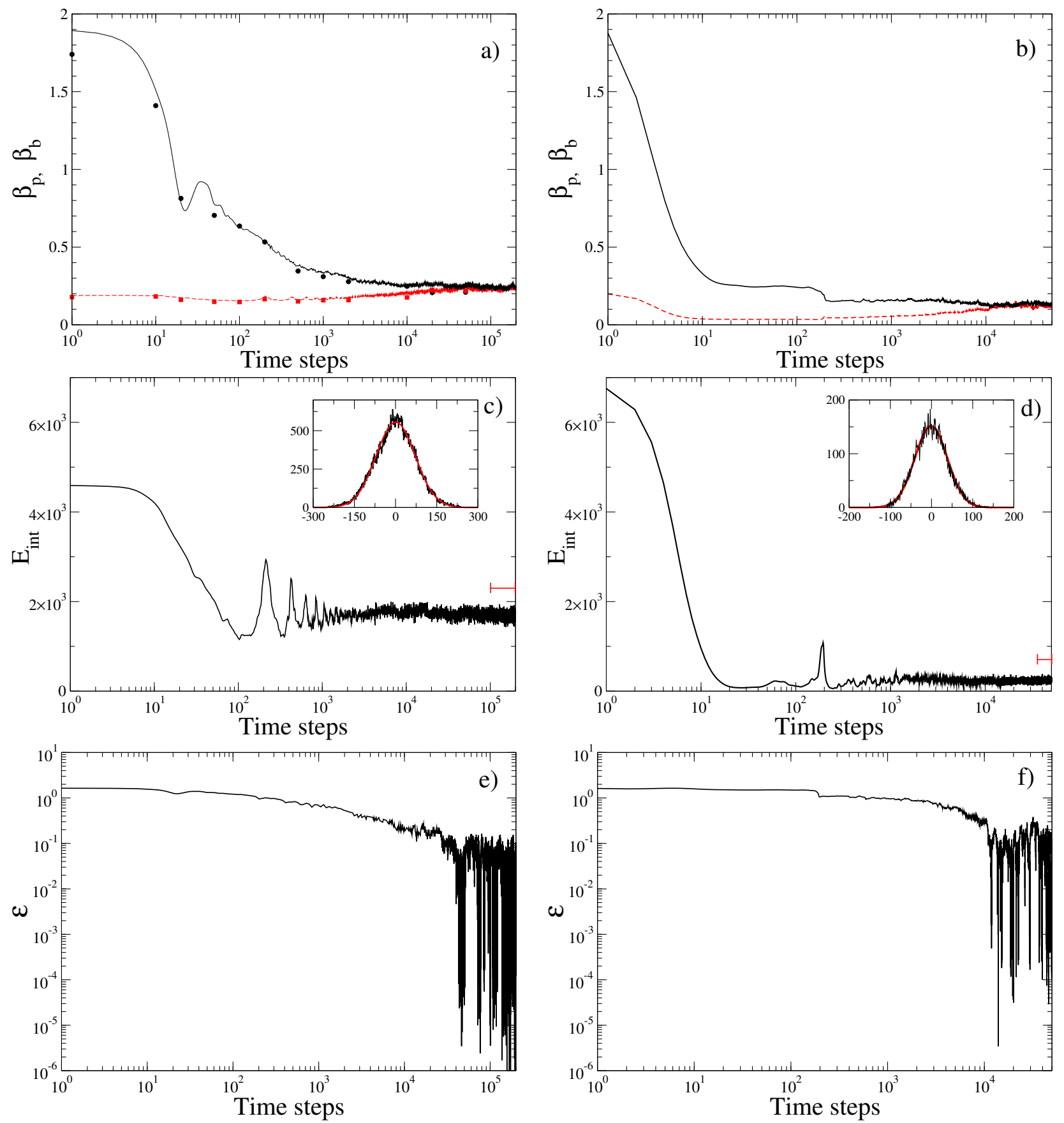

FIG. 2: Thermalization in one and three dimensions. (a) Evolution of inverse temperature with time for the two species in 1D, the cold bath particles ( $\beta_{b}$, continuous black curve) and the warm target particles ( $\beta_{p}$, dashed red curve), obtained by single run simulations. The parameters are the same as in Fig. 5 b in Ref. [16] $\left(\gamma_{E}=0.1, \lambda=0.1, N_{p}=N_{t}=10^{3}, m=m_{0}=1.0, \omega=1.0\right.$ and $\Omega=144 / 89$ ), and the dots represent the data reported in the latter paper using a fitting procedure for the energy distribution of the baths at specific times, while the line is the resulting curve from the determination of the inverse temperature through evaluation of the average energy and the average squared energy at each time step using Eq. (6). Panel (b) shows that the same metric can be also used to track thermalization in the full $3 \mathrm{D}$ case, for $\gamma_{E}=14, \lambda=0.5, N_{p}=N_{b}=200$. Panels (c) and $(\mathrm{d})$ show the evolution of the total interaction energy with time corresponding to (a) and (b), respectively. In both cases, the episodic interactions between the two species get increasingly random with time until it becomes a noisy, nearly constant value. The insets show the distribution of fluctuations around the constant value which is clearly well-described by Gaussians (red curves in the insets), evaluated over the intervals indicated by the horizontal segment in the the bottom-right part of the main figures. Panels (e) and (f) show the time-dependence of the parameter $\epsilon$ and its effectiveness in quantifying the onset of thermalization. 


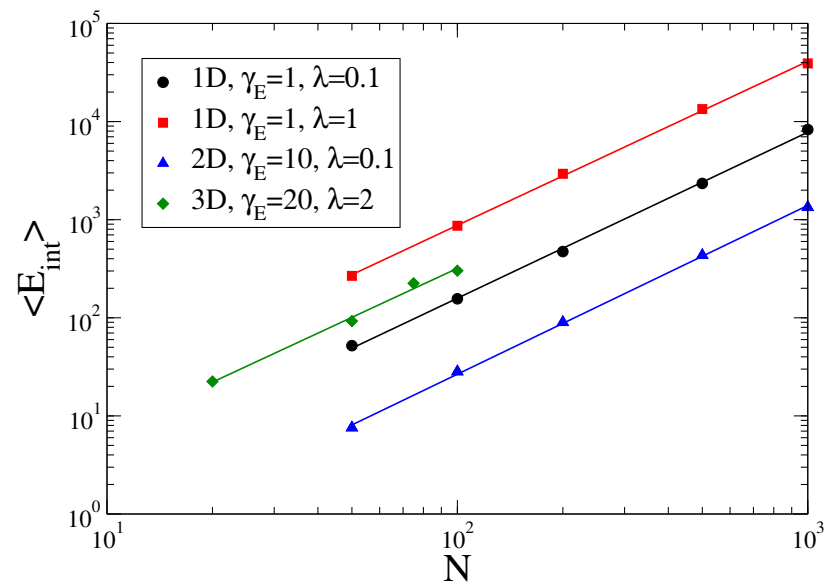

FIG. 3: Scaling of the long time averaged total interaction energy in $1 \mathrm{D}, 2 \mathrm{D}$ and $3 \mathrm{D}$ versus the system size, for equal number of particles in the two systems, $N_{b}=N_{p}=N$. Two cases are shown in 1D corresponding to different values of the parameter $\lambda$. The time interval over which the average is taken is chosen based on the $\epsilon$ parameter with a criterion $\epsilon \leq 10^{-4}$. In the $1 \mathrm{D}$ cases, the average is taken over a longer time than in the higher dimensionality cases, i.e. from $80,000-100,000$ time steps. The power-law fits yield values of the exponent equal to $\alpha=(1.69 \pm 0.03),(1.67 \pm 0.02),(1.71 \pm$ $0.03),(1.65 \pm 0.09)$ for the corresponding cases discussed in the label from top to bottom respectively. It should be noted that the suggested exponent of $5 / 3$ is expected in the large $N$ limit and when thermalization is well-established. In higher dimensional dynamics, the combination of smaller $N$ and incomplete thermalization results in the largest errors.

therefore changes in the interaction energy over time will result in changes in the correlation function. This may be expressed as

$$
\frac{d C_{b p}}{d t}=-\frac{\lambda^{2}}{\left\langle E_{\text {int }}\right\rangle} \frac{d\left\langle E_{\text {int }}\right\rangle}{d t} .
$$

Contrasting this expression with definitions used for effective temperatures suggests that the total interaction energy $\left\langle E_{\text {int }}\right\rangle$ here plays the role of $k_{B} T_{\text {eff }}$ in 25,27 . This relationship also makes clear that stationarity of the correlation function results in the stationarity of the total interaction energy and, hence, the effective temperature.

Before moving to a more detailed discussion of the inferences seen in the behaviour of the interaction energy with time, we draw attention to Figs. 2(e) and (f) which show a simple but seemingly effective way of quantifying the thermalization time. What is shown is the quantity

$$
\epsilon=2 \frac{\left|\beta_{p}-\beta_{b}\right|}{\beta_{p}+\beta_{b}}
$$

which relates the absolute difference in $\beta$ between the two species with their mean value. Contrasting the thermalization plots in Fig. 2(a) and (b) with the $\epsilon$ behaviour clearly suggests the possible use of this measure to numerically quantify thermalization times. Notice that when the $\epsilon$ parameter drops below a given threshold, say $\epsilon \leq 10^{-3}-10^{-4}$, the number of spikes with values lower than the threshold increases significantly even if occasionally the parameter raises again to higher values. These fluctuations in the thermalization dynamics are more clearly visible in $\epsilon$ than in the inverse temperature curves. The spikes are correlated to the details of the thermalization dynamics, and in the absence of a damping mechanism can last for long times. Their duration is inversely proportional to the instantaneous rates when the two $\beta$ curves cross each other. Inverse temperatures which are very close to each other for a large extent of time therefore results in a broad time interval below threshold. Additionally, we expect the finite number of particles to also play a role with $\epsilon$ being a more effective metric with increasing $N_{b}, N_{p}$, one which could be useful in quantitatively contrasting cooling strategies.

Returning to the interaction energy, a noisy plateau is seen for longer times coinciding with the thermalization of the two species. In this time domain, the timeaveraged mean value can be computed and the distribution of fluctuations about this mean constructed. This is shown in the insets in Figs. 2(c) and (d) and both are clearly Gaussian. Thus the total energy (Hamiltonian) which was originally distributed as $H_{p}+H_{b}+H_{\text {int }}$ evolves with time to the approximation $H_{p}^{\prime}+H_{b}^{\prime}+G(t)+$ const where $G(t)$ represents the Gaussian fluctuations in the interaction energy. As the interaction energy was connected to an effective $\beta$, these can be viewed as akin to thermal fluctuations. This is also an indicator of the onset of thermalization and we recover the textbook, nonor weakly interacting construct used when defining macro variables such as temperature. As seen from Fig. 2, this behaviour is seen irrespective of the dimensionality of the dynamics suggesting a universal mechanism for thermalization. The energy content in the mean (constant) value, as contrasted with that given by the initial temperatures $T_{p}$ and $T_{b}$ of the two subsystems, determines whether or not the final equilibrium temperature is hotter than either of two initial ones, a point which we will return to later. We note here that, as a consequence, varying the strength of the inter-species interaction can be used as a control parameter to achieve specified final states [28]. We anticipate that the exact functional form of the interaction only affects the timescales for thermalization rather than the universal behaviour itself. The expectation is based on the time evolution of the interaction energy which transitions from deterministic interactions between the two species, based clearly on the details of the dynamics, to a probabilistic regime described by an average interaction per particle with small fluctuations. This suggests homogenization of the neighbourhood around each test particle, meaning that the two atomic species are well-mixed. This is a view which is consistent with another result seen in the numerics, suggestive of a possible connection to a result from fluid dynamics.

We consider the time-averaged total interaction energy as a function of the system size (number of oscillators) 

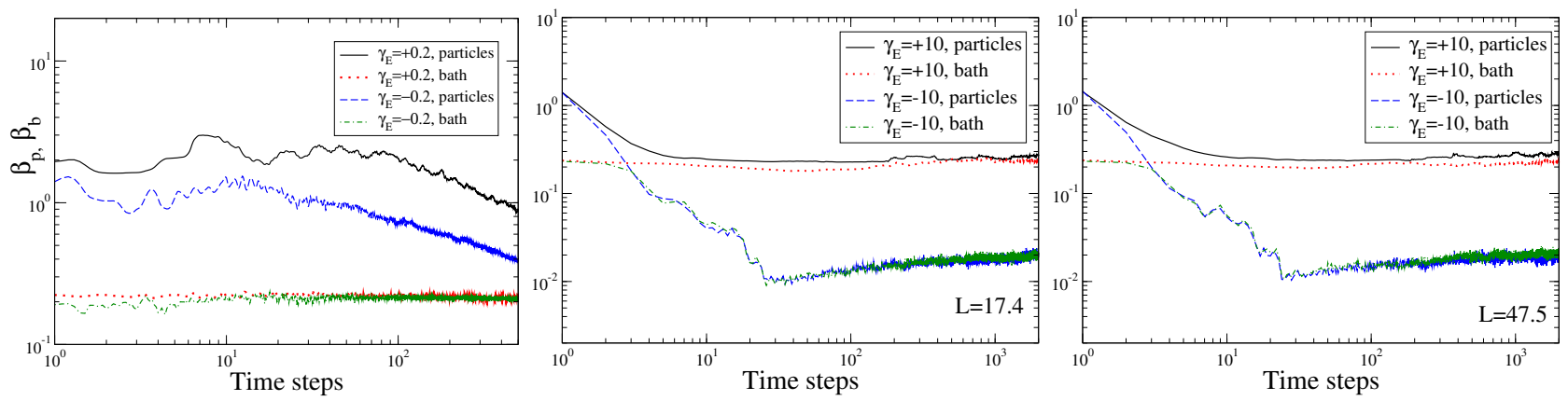

FIG. 4: Comparison between the dynamics of thermalization in (a) 1D where the only difference is in the sign of the coupling strength $\gamma_{E}$. (b) and (c) are the analogous effects explored in 3D where the difference between the two plots is the total angular momentum of the two-species system which affects the number of inter-species encounters, as indicated in the lower right corner of each plot.

where $N_{b}=N_{p}=N$. This was done both for one- and higher dimensional dynamics. In each case, the parameter values considered resulted in thermalization over the total time scale considered. We note that for higher dimensions, a larger interaction strength was considered to ensure thermalization over a shorter timescale, simply for numerical considerations. The results, as seen from Fig. 3, display power-law scaling with system size with a common exponent (within fitting errors) independent of dimensionality. In all instances, the time averaged interaction energy scales as $N^{\alpha}$ where $\alpha$ is close to $5 / 3$, an exponent which has significance in another context.

This value of the scaling exponent is reminiscent of the Kolmogorov scaling, as $k^{-5 / 3}$ where $k$ is the wavenumber, associated with turbulent mixing [29, 30]. In that

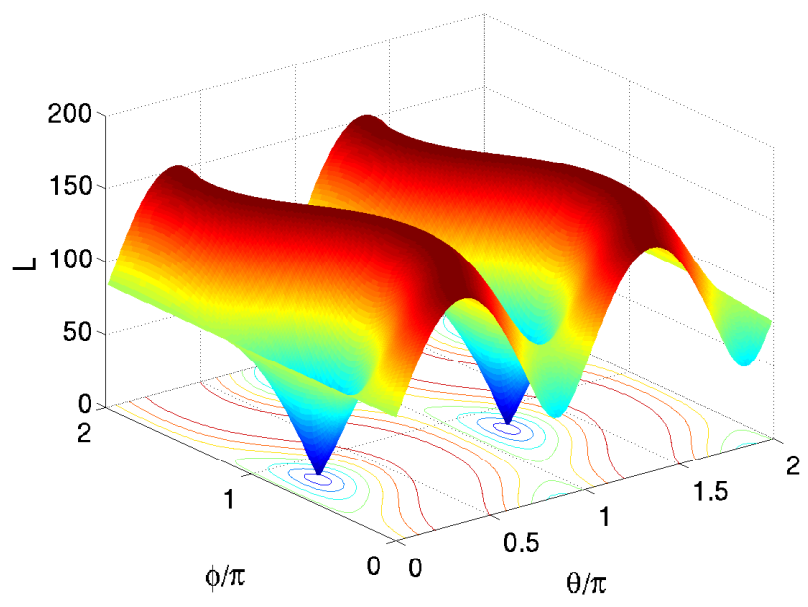

FIG. 5: Total angular momentum of the atomic clouds versus the angles $\theta$ and $\phi$ between the momentum and the position of each particle for given inverse temperatures $\beta_{p}=0.2$ and $\beta_{b}=2.0$. The behaviour is identical when considering the differential angular momentum of the atomic clouds. The profile shown does depend on the particle numbers in each species and here we use $N_{b}=N_{p}=500$. case, this value can be recovered using elegant, dimensional arguments involving viscosity and an energy transfer rate. In our situation, the inter-species interaction is responsible for both energy transfer as well as an 'effective damping' mechanism. Nevertheless, we speculate that the mechanism for eliminating thermal heterogeneity is analogous to the removal of density or thermal gradients in the fluid problem. Here, we move from a deterministic description to a statistical one as evidenced by the behaviour of the interaction energy. Notice that, while the Caldeira-Leggett model allows for thermalization, due to the linear nature of the interatomic interactions there is no energy transfer between different timescales, despite the presence of a continuum of frequencies in the thermal bath. Here instead we expect mixing among all timescales due to the nonlinear interaction, in spite of the monochromatic baths. This occurs in a manner similar to the mixing of different lengthscales in the turbulent mixing of fluids. A more detailed exploration of this intriguing analogy is ongoing. In the current context, we consider the scaling result another quantitative indicator of universal aspects of the thermalization mechanism.

\section{SINGULAR ASPECTS OF HIGHER-DIMENSIONAL THERMALIZATION}

It is clear that the dynamics of thermalization in higher dimensions depends on aspects that are not present in the simpler 1D case. Significant amongst these is angular momentum which brings with it the ability to avoid interactions, notably head-on collisions, thus inhibiting energy exchange which, in turn, slows down the thermalization process. Of course, changing the nature of the interaction from repulsive to attractive brings in aggregation mechanisms which force interactions.

We begin by studying the dependence of the dynamics on the sign of the interaction potential. As shown in Fig. 4(a), the use of attractive interactions results in 


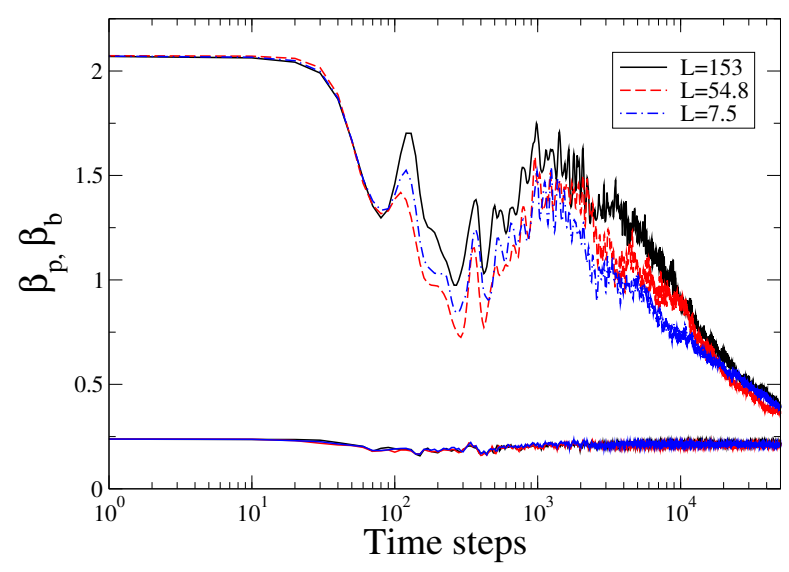

FIG. 6: Comparison between thermalization trajectories for initial conditions differing only by the amount of total angular momentum, with $N_{b}=N_{p}=500, \gamma_{E}=-0.1$ and $\lambda=1.0$ in all cases. The total angular momentum values are indicated in the legend. The low value of $\gamma_{E}$ has been chosen to emphasize differences due to total angular momentum.

a faster thermalization in $1 \mathrm{D}$ with respect to the repulsive interaction with the same absolute value of $\gamma_{E}$. This can be easily interpreted from the propensity for the two clouds to cluster in the former case. In an experimental scenario, however, this effect will be accompanied by larger three-body decay rates for attractive interactions also due to the higher densities, with consequent increases in heating rates. The simulations in $3 \mathrm{D}$, for the same range of parameters used in $1 \mathrm{D}$, show that thermalization in general seems to occur, if at all, on considerably longer timescales. This may be interpreted in configuration space as due to the presence of a multitude of 'avoiding' trajectories, and of a partial overlap between the two atomic clouds. Of course, increasing the interaction strength $\gamma_{E}$ or the range $\lambda$ or the sign of the interaction are all ways to speed up the process. Figure 4(b) shows the role of larger $\left|\gamma_{E}\right|$ in achieving this.

Additionally, as the particles also possess angular momentum, they are not forced as in the 1D case to have $s$ wave-like encounters with the other particles. Therefore, all other conditions for the atomic clouds being the same, we expect slower thermalization in higher dimensions. Angular momentum is a meaningful quantity to be considered in this setting, as both the harmonic trapping potential and the interspecies interaction are central forces, therefore the total angular momentum available in the mixture is conserved. In principle, the Boltzmann distribution of each cloud depends on all independently conserved quantities, including angular momentum (see supplementary information in 31 for a detailed discussion), but thermalization leads to a stationary state in which its role is expected to be marginal. In order to quantify these hypotheses we have injected a controllable total angular momentum to the initial conditions of the two clouds, to see how the thermalization timescale depends on it. The

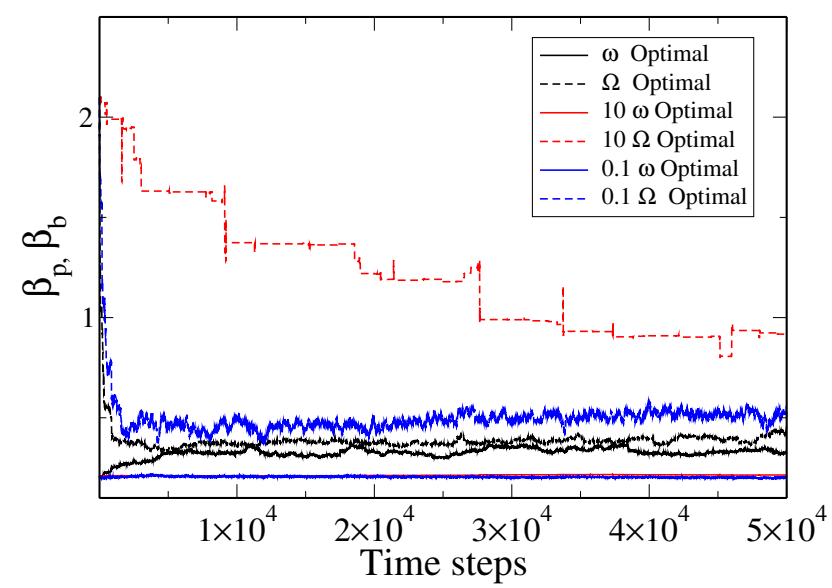

FIG. 7: The effect of changing the frequency ratio $\Omega / \omega$. As indicated in the legend, the optimal ratio suggested in the text and significantly smaller and larger values are shown. In all cases, the other parameters are $T_{b}=0.5, T_{p}=5.0, N_{p}=$ $N_{b}=200, \gamma_{E}=14$ and $\lambda=0.1$.

initialization of the two baths must be rearranged in such a way that we control the amount of angular momentum energy. The Hamiltonian for a single particle in spherical coordinates is $H=m \dot{r}^{2} / 2+m \omega^{2} r^{2} / 2+L^{2} /\left(2 m r^{2}\right)$, where the first two terms represent the radial kinetic energy and the harmonic potential energy, respectively, and the third contains the kinetic energy due to the presence of angular momentum. This is implemented by first picking an energy value for each particle consistent with the Boltzmann distribution and random positions from which the magnitude of the total momentum can be computed. The choice of the direction of momentum with respect to the vector $\overrightarrow{\mathbf{r}}$ can then be controlled by means of two angles, $\theta$ and $\phi$, such that $p_{x}=p \sin \theta \cos \phi, p_{y}=p \sin \theta \sin \phi, p_{z}=p \cos \theta$. In Fig. 5 . we show the dependence of the total angular momentum of the two clouds as a function of $\theta$ and $\phi$, chosen to have the same value for each atom. The initial temperatures of both clouds are specified in the caption. The angular momentum differential between the two clouds shows identical dependence on the two angles.

The control of the angular momentum of the two clouds allows us to compare thermalization in various cases. We return to panels (b) and (c) of Fig. 4 which is a situation where the interaction strength is strong. The only difference between the two panels is the total angular momentum of the two clouds. Here, we expect the interaction strength to dominate and the role of the angular momentum is barely visible. In order to isolate any difference arising strictly from changes in the total angular momentum, we reduce the value of $\gamma_{E}$. These results are shown in Fig. 6 where we compare three cases, minimal and maximal angular momentum, and an intermediate value. Any sensitivity to angular momentum is apparent only at an intermediate stage, starting af- 
ter about $10^{2}$ time steps, and lasting about two decades. In the region around $10^{3}$ time steps the three curves of the lower temperature bath, in spite of statistical fluctuations, settle themselves in a systematic order approaching equilibrium with the hotter bath according to the content of angular momentum, and with a thermalization speed inversely proportional to the latter. Beyond about $2 \times 10^{4}$ time steps the difference among the curves is negligible. This is not too surprising given the earlier discussion on the universal route to thermalization. Changes in total angular momentum should only affect the deterministic phase of the thermalization process which, given the many-particle and nonlinear nature of the interaction, can be quite short. Experimentally, control of the angular momentum of a Bose-Einstein condensate has been demonstrated via coherent transfer from optical fields using a stimulated Raman process [32, providing a proto$\mathrm{col}$ for testing these findings. As commented on earlier, we only analyse the case of velocity-independent interactions throughout this contribution, unlike the analysis reported in [16. We expect that further differences in thermalization dynamics could arise by using velocitydependent interactions due to the peculiar role of the velocity distribution in assigning angular momenta.

Long-time simulations show that the approach to complete equilibrium is rather slow even for moderate values of interaction strength. A more sensitive (and controllable) knob which can be used to speed up thermalization is available by optimizing the spatial overlap between the two clouds. The unavoidable presence of atoms with large angular momentum and high energy in the Boltzmann tail will make these atoms very weakly interacting with the rest of the cloud of the other species. Therefore, no matter of how long one waits for thermalization, these atoms will form a sort of 'closed subsystem' at nearly constant energy, apart from occasional close encounters in which large amounts of energy will be exchanged. This can be mitigated if the overlap between the clouds is maximized at all times. The average squared position of the atoms of each atomic species with respect to the minimum of the trapping potential is written in terms of the corresponding temperature as $\left\langle q^{2}\right\rangle=2 k_{B} T_{b} / m \omega^{2}$ and $\left\langle Q^{2}\right\rangle=2 k_{B} T_{p} / M \Omega^{2}$. It is therefore possible to equate the mean squared positions for the two clouds if

$$
\frac{\Omega}{\omega}=\left(\frac{m}{M} \frac{T_{p}}{T_{b}}\right)^{1 / 2} .
$$

In order to have optimal overlap, one should continuously change the trapping frequency ratio in accordance with this expression. This possibility, discussed in 33 (in particular see Fig. 6 there), restates the problem in the context of a control theory approach.

Here we envisage a much simpler strategy in which a single trapping frequency ratio is chosen based on the initial temperatures. The results are shown in Fig. 7 where the thermalization curve for the optimal frequency ratio is contrasted with those corresponding to smaller and larger ratios. The increased efficiency at the optimal ra- tio is quite apparent. We have not explored this further as yet though the usefulness would be most apparent in a well-defined experimental context. We do note here that it is crucial to choose this ratio in such a way that the optimal overlap occurs at later times, when the initial drop in temperature of the hottest cloud, occurring regardless of sophisticated optimizations, has already taken place.

\section{NUMBER ASYMMETRY AND CENTER OF MASS MODE-LOCKING}

Thus far, we have only presented situations where the two species have equal numbers of particles. If this condition is upset, interesting phenomena occur as the species with larger number of atoms (the 'majority species' thereafter) will lead the thermalization based on the dominance of its heat capacity with respect to the species with smaller number of atoms (the 'minority' species). As long as the nondegenerate limit is considered for small interaction strength, this situation is all contained in the Dulong-Petit law with no surprising implications. The case of strong coupling is not quite so trivial. Here we have to include the ensemble averaged interaction energy in our estimate of the final thermalized temperature, leading to

$$
T_{e q}=\frac{N_{p} T_{p}+N_{b} T_{b}}{N_{p}+N_{b}}+\frac{\left\langle E_{\mathrm{int}}\right\rangle}{\left(N_{p}+N_{b}\right) k_{B}} .
$$

In the case of strong coupling and equal numbers, it is clear that the final temperature can exceed both of the initial values. This was noted both in Ref. [16] and seen in some of the results here as well, notably Fig. 2(b) and Fig. 4(b)/(c). Even if the final temperature lies between the initial ones, the interaction energy needs to be included in the calculation of the final temperature, which can be quantitatively verified from Fig. 2(a). However, where the species numbers are strongly asymmetric, $N_{p}<<N_{b}$, the effect of the interaction term is greatly suppressed, reducing it to a weak coupling situation. We note also that minority gases, even for the extreme case of single atoms, embedded in majority gases are also crucial for precise and accurate thermometry of ultracold gases [34 36].

A second phenomenon worthy of attention for numberasymmetric clouds is the presence of 'mixing' in the frequency spectrum of the oscillations. This may be related to recent measurements on the relative motion of two components of an ultracold Fermi-Bose ${ }^{6} \mathrm{Li}^{7} \mathrm{Li}$ trapped mixture with asymmetric numbers of atoms. Their results indicate a shift in the oscillation frequency of the minority species ${ }^{7} \mathrm{Li}$ while the majority species ${ }^{6} \mathrm{Li}$ is largely unaffected [37, 38. The phenomenon has been quantitatively addressed for the case of a superfluid mixture by considering the Bose atoms as impurities immersed in the Fermi superfluid. Adding a mean-field interaction term to the external trapping potential, neglecting the back-action of the bosons on the fermions, 

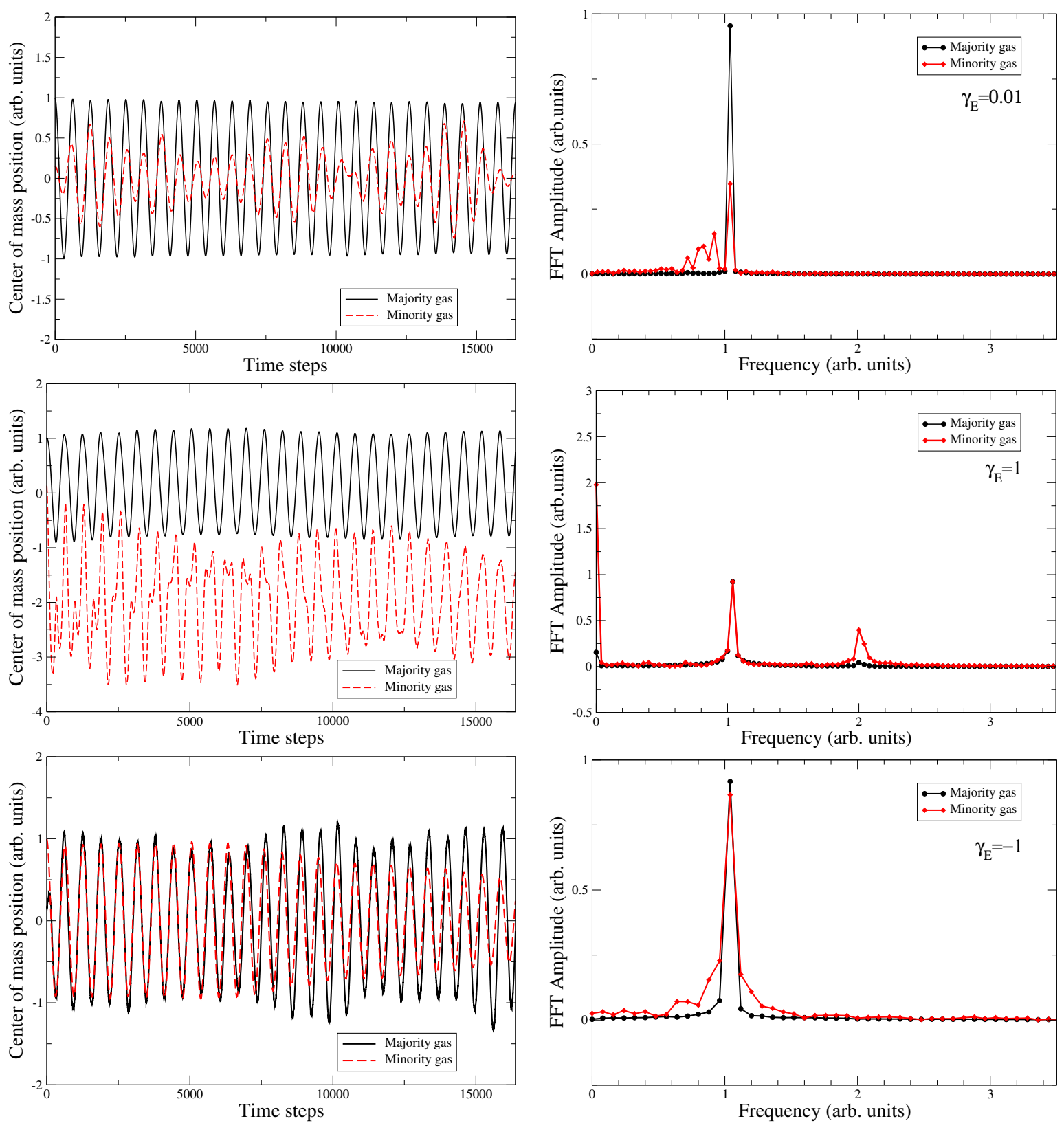

FIG. 8: (Color online) Case of $1 \mathrm{D}$ dynamics with asymmetric particle numbers with $N_{b}=1000, N_{p}=100$. $\omega=1 ; \Omega=0.9$, $\beta=1$ and $\lambda=0.1$. The values of $\gamma_{E}$ is specified in the figures. Panels (a), (c) and (e) show the time evolution of the centers of mass of the majority cloud (black, continuous lines) and the minority cloud (red, dashed lines) while (b), (d) and (f) are the corresponding FFTs (black, dot points, and red, diamonds, respectively) over the duration shown in the left panels $\left(2^{14}\right.$ time steps).

and using a local-density approximation, an effective potential was obtained. This led to a trapping frequency of $\tilde{\omega}_{b}=2 \pi \times 14.97 \mathrm{~Hz}$, in agreement (within two standard deviations) with the measured value of $\tilde{\omega}_{b}^{e x p}=2 \pi \times 15.00(2) \mathrm{Hz}$, and with the experimental value of the trapping frequency of ${ }^{7} \mathrm{Li}$ in the absence of ${ }^{6} \mathrm{Li}$ atoms $\tilde{\omega}_{b}^{\text {exp }}=2 \pi \times 15.27(1) \mathrm{Hz}$. A second effect was observed above the critical temperatures for superfluidity for both gases, at $T \simeq 0.34 T_{F}, \simeq T_{c B}$, with $T_{F}$ the Fermi temperature and $T_{c B}$ the critical temperature for BoseEinstein condensation (coinciding with the critical temperature for Bose superfluidity), in which ${ }^{7} \mathrm{Li}$ oscillates at the same frequency of ${ }^{6} \mathrm{Li}$ (also equaling the damping constant) [38. In the same paper, a qualitative description was proposed in terms of a "sort of quantum Zeno effect" (see details in the supplementary informations of Ref 38) related to the violation, due to mean-field effects, of the Kohn theorem [39] for dipole oscillations. 
Our analysis starts by considering two atomic clouds confined around the same location but, in general, with different trapping frequencies. The temperatures of the clouds are set to be equal, and the center of mass of one of the two clouds is initially offset by a given amount. The two clouds have different number of atoms with the mismatch given by $N_{2} / N_{1}=10$ such that we can identify a 'minority' species and a 'majority' species. Their intrinsic trapping frequencies are chosen to differ by $10 \%$. Whenever $\gamma_{E} \neq 0$ the two clouds will interact, modifying the dynamics of their centers of mass. Figure 8(a) shows a case for small coupling strength $\left(\gamma_{E}=10^{-2}\right)$ of repulsive character. A more quantitative assessment is achieved by taking the Fast Fourier Transform (FFT) spectrum of the centers of mass motion, as shown in Fig. 8(b). While the FFT spectrum of the majority cloud peaks at a constant value equal to the one in the absence of interactions, the corresponding one of the minority cloud has various components, with the highest peak occurring at the frequency of the majority cloud. This is effectively a "mode locking" phenomenon. Although the analysis is carried out for a $1 \mathrm{D}$ system, no further qualitative insights should be gained by analyzing a full three-dimensional situation, at least in the nondegenerate regime.

If the interspecies interaction is boosted by two orders of magnitude, the minority cloud center of mass locks into the frequency of the majority cloud more quickly. Though visible in Fig. 8(c), it is more readily seen in the near coincident peaks in FFT shown in Fig. 8(d), even in amplitude, with little components at smaller frequencies. Notice that this occurs even if, due to the strong repulsive interaction, the centers of mass of the two clouds never intersect each other, as evident in Fig. 8(c). The FFT analysis also shows an intriguing feature, the presence of a second harmonic. This can be explained by the intrinsic nonlinear nature of the interaction term. By Taylor-expanding this term in the difference between two coordinates, we notice that next to the quadratic term in $\xi_{n m}=\left(q_{n}-Q_{m}\right) / \lambda$ is a quartic term in the same variable. This term is relevant in the strong coupling regime (large $\gamma_{E}$ ) and when the expansion term $\xi_{n m}$ is large, i.e. if the average separation between two atoms of the two clouds is large and/or the interaction range $\lambda$ is small. Under these circumstances second harmonic generation becomes favourable and could result in precision assessment of nonlinearities in the interaction between species. Notice that strong interactions alone are not a sufficient condition. If the interaction is attractive, for the same $\gamma_{E}$ used in the repulsive case, we see from Fig. 8(e) and (f) that there is no second harmonic generation in spite of a nearly complete overlap between the centers of mass of the two clouds.

\section{CONCLUSIONS}

Motivated by sympathetic cooling of atomic gases, we have extended our exploration of a modified onedimensional Caldeira-Leggett model to higher dimensions in the nondegenerate regime. This process isolated some universal characteristics which we argue are largely independent of the details of the interspecies interaction. The basis for this inference is the transition of the thermalization dynamics from a deterministic regime to one described by statistical measures. However, the dynamical details are relevant for describing transients which are important in the design of optimal sympathetic cooling protocols. In the process, we also suggest a metric for numerically determining the onset of thermalization which may be useful for quantifying the efficiency of contrasting strategies. We note that there are other recent experimental contexts, such as the high-resolution observation of Brownian motion in optical tweezers [40], where the universal scaling aspects of thermalization discussed here may be directly tested.

We have also explored the dependence of thermalization on the nature of the interaction as well as parameters specifically pertinent to higher dimensional dynamics, notably angular momentum. It should be noted that we have encountered cases in 3D where, for strong interactions and as a consequence of angular momentum mismatch between the two clouds, the onset of thermalization is significantly delayed. The principal reason for this behaviour appears to be marginal overlap between the evolved clouds after some initial energy exchange. We envision revisiting these situations by using the center of mass dynamics of each cloud to verify if this is, in fact, the mechanism. Here, we have illustrated the value of considering the center of mass dynamics by identifying a mode-locking mechanism relevant to recently reported experiments on Bose-Fermi mixtures in the normal regime.

In our model, this mode-locking results from nonlinearities in the interspecies interactions. In realistic experimental setups, nonlinearities may also arise from inherent aspects of the trapping potential which can, in general, enhance the effects due to interactions. This is particularly manifest in optical dipole traps [41, which will be the focus of future work especially with regard to their thermalization efficiency of cold atomic samples in the nondegenerate regime.
[1] I. Bloch, J. Dalibard, and W. Zwerger, Many-body physics with ultracold gases, Rev. Mod. Phys. 80, 885, (2008).

[2] M. Lewenstein, A. Sampera, and V. Ahufinger, Ultracold atoms in optical lattices, (Oxford University Press, 2012).

[3] J. Mur-Petit, A. Polls, M. Baldo, H.-J. Schulze, Pairing in two-dimensional boson-fermion mixtures, Phys. Rev. A 69, 023606 (2004). 
[4] M. Dalmonte, K. Dieckmann, T. Roscilde, C. Hartl, A. E. Feiguin, U. Schollwöck, F. Heidrich-Meisner, Dimer, trimer, and Fulder-Ferrell-Larkin-Ovchinnikov liquids in mass- and spin-unbalanced trapped binary mixtures in one dimension, Phys. Rev. A 85, 063608 (2012).

[5] T. V. Barmashova, K. A. Martiyanov, V. B. Makhalov, and A. V. Turlapov, Fermi liquid-to-Bose condensate crossover in a two-dimensional ultracold gas experiment, Ups. Fiz. Nauk 186, 183 (2016) [Phys. Usp. 59, 174 (2016)].

[6] D. C. McKay and B. DeMarco, Cooling in strongly correlated optical lattices: prospects and challenges, Rep. Prog. Phys. 74, 054401 (2011).

[7] R. Onofrio, Cooling and thermometry of atomic Fermi gases, Phys. Usp. 59, 1129 (2016); Usp. Fiz. Nauk 186, 1229 (2016).

[8] V. B. Magalinskii, Dynamical model on the theory of the Brownian motion, Sov. Phys. JETP 9, 1381 (1959).

[9] P. Ullersma, An exactly solvable model for Brownian motion. 1. Derivation of Langevin equation, Physica 52, 27 (1966).

[10] P. Ullersma, An exactly solvable model for Brownian motion. 2. Derivation of Fokker-Planck equation and master equation, Physica 32, 56 (1966).

[11] P. Ullersma, An exactly solvable model for Brownian motion. 3. Motion of a heavy mass in a linear chain, Physica 32, 74 (1966).

[12] P. Ullersma, An exactly solvable model for Brownian motion. 4. Susceptibility and Nyquist's theorem, Physica 32, 90 (1966).

[13] A. O. Caldeira and A. J. Leggett, Influence of dissipation on quantum tunneling in macroscopic systems, Phys. Rev. Lett. 46, 211 (1981).

[14] A. O. Caldeira and A. J. Leggett, Quantum tunnelling in a dissipative system, Ann. Phys. 149, 374 (1983).

[15] A. O. Caldeira and A. J. Leggett, Influence of damping on quantum interference: An exactly soluble model, Ann. Phys. 149, 374 (1983).

[16] R. Onofrio and B. Sundaram, Effective microscopic models for sympathetic cooling of atomic gases, Phys. Rev. A 92, 033422 (2015).

[17] K. Schönhammer, Thermodynamics and occupation numbers of a Fermi gas in the canonical ensemble, Am. J. Phys. 68, 1032 (2000).

[18] W. J. Mullin and J. P. Fernandez, Bose-Einstein condensation, fluctuations, and recurrence relations in statistical mechanics, Am. J. Phys. 71, 661 (2003).

[19] S. T. Smith and R. Onofrio, Thermalization in open classical systems with finite heat baths, Eur. Phys. J. B 61, 271 (2008).

[20] P. Hanggi, G.-L. Ingold, and P. Talkner, Finite quantum dissipation: the challenge of obtaining specific heat, New J. Phys. 10, 115008 (2008).

[21] H. Hasegawa, Classical small systems coupled to finite baths, Phys. Rev. E 83, 021104 (2011).

[22] A. Carcaterra and A. Akay, Fluctuation-dissipation and energy properties of a finite bath, Phys. Rev. E 84, 011121 (2011).

[23] H. Hasegawa, Specific heat anomalies of small quantum systems subjected to finite baths, J. Math. Phys. 52, 123301 (2011).

[24] H. Hasegawa, Responses to applied forces and the
Jarzynski equality in classical oscillator systems coupled to finite baths: An exactly solvable non-dissipative nonergodic model, Phys. Rev. E 84, 011145 (2011).

[25] L. F. Cugliandolo, J. Kurchan, and L. Peliti, Energy flow, partial equilibration, and effective temperatures in systems with slow dynamics, Phys. Rev. E 55, 3898 (1997).

[26] L. Berthier, J. L. Barrat, and J. Kurchan, Response function of coarsening systems, Eur. Phys. J. B 11, 635 (1999).

[27] T. Speck and U. Seifert, Restoring a fluctuationdissipation theorem in a nonequilibrium steady state, Europhys. Lett. 74, 391 (2006).

[28] A. Rahmani, T. Kitagawa, E. Demler, and C. Chamon, Cooling through optimal control of quantum evolution, Phys. Rev. A 87, 043607 (2013).

[29] A. N. Kolmogorov, The local structure of turbulence in incompressible viscous fluid for very large Reynolds numbers, Proceedings of the USSR Academy of Sciences 30, 299 (1941).

[30] A. N. Kolmogorov, Dissipation of energy in the locally isotropic turbulence, Proceedings of the USSR Academy of Sciences 32, 16 (1941).

[31] D. Guéry-Odelin, J. G. Muga, M. J. Ruiz-Montero, and E. Trizac, Nonequilibrium solutions of the Boltzmann equation under the action of an external force, Phys. Rev. Lett. 112, 180602 (2014).

[32] K. C. Wright, L. S. Leslie, and N. P. Bigelow, Optical control of the internal and external angular momentum of a Bose-Einstein condensate, Phys. Rev. A 77, 041601(R) (2008).

[33] M. Brown-Hayes, Q. Wei, C. Presilla, and R. Onofrio, Thermodynamical approaches to efficient sympathetic cooling in ultracold Fermi-Bose atomic mixtures, Phys. Rev. A 78, 013617 (2008).

[34] C. Tuchendler, A. M. Lance, A. Browaeys, Y. R. P. Sortais, and P. Grangier, Energy distribution and cooling of a single atom in an optical tweezer, Phys. Rev. A 78, 033425 (2008).

[35] C. Sabín, A. White, L. Hackermuller, and I. Fuentes, Impurities as a quantum thermometer for a Bose-Einstein condensate, Sci. Rep. 4, 6436 (2014).

[36] M. Hohmann, F. Kindermann, T. Lausch, D. Mayer, F. Schmidt, and A. Widera, A single atom thermometer for ultracold gases, Phys. Rev A 93, 043607 (2016).

[37] I. Ferrier-Barbut, M. Delehaye, S. Laurent, A. T. Grier, M. Pierce, B. S. Rem, F. Chevy, and C. Salomon, A mixture of Bose and Fermi superfluids, Science 345, 1035 (2014).

[38] M. Delehaye, S. Laurent, I. Ferrier-Babut, S. Jin, F. Chevy, and C. Salomon, Critical velocity and dissipation of an ultracold Bose-Fermi counterflow, Phys. Rev. Lett. 115, 265303 (2015).

[39] W. Kohn, Cyclotron Resonance and de Haas-van Alphen Oscillations of an Interacting Electron Gas, Phys. Rev. 123, 1242 (1961).

[40] T. Li, S. Kheifets, D. Medellin, and M. G. Raizen, Measurement of the instantaneous velocity of a Brownian particle, Science 328, 1673 (2010).

[41] C. S. Adams, H. J. Lee, N. Davidson, M. Kasevich, and S. $\mathrm{Chu}$, Evaporative cooling in a crossed dipole trap, Phys. Rev. Lett. 74, 3577 (1995). 